\title{
A NOTE ON NONSMOOTH OPTIMAL CONTROL PROBLEMS
}

\author{
Hans Joachim Oberle \\ Department of Mathematics, University of Hamburg, Germany \\ oberle@math.uni-hamburg.de
}

Keywords: nonsmooth optimal control, necessary conditions, singular state subarcs

\section{INTRODUCTION}

The lecture is concerned with general optimal control problems (OCP) which are characterized by a nonsmooth ordinary state differential equation. More precisely, we assume that the right-hand side of the state equation is piecewise smooth and that the switching points, which separate these pieces, are determined as roots of a state- and control-dependent (smooth) switching function.

Nonsmooth optimal control problems of this type rarely have been mentioned in the literature. Of course, they are special examples for the rather general theory of Clarke, Ref. (1).

For this kind of nonsmooth OPC new necessary conditions are derived. These conditions depend on the order of the switching function with respect to the control variable and of certain regularity assumptions concerning the occurence of subarcs where the switching function vanishes identically, so called singular subarcs. Examples of order zero and of order one are considered.

\section{NONSMOOTH OCPs}

The general nonsmooth OCP is given as follows. Problem (OCP). Determine a piecewise continuous control function $u:[a, b] \rightarrow \mathbb{R}^{m}$, such that

$$
I=g(x(b))
$$

is minimized subject to the following constraints (state equations, boundary conditions, and control constraints)

$$
\begin{aligned}
& x^{\prime}(t)=f(x(t), u(t)), \quad t \in[a, b], x(t) \in \mathbb{R}^{n}, \\
& r(x(a), x(b))=0, \\
& u(t) \in U=\Pi_{i}\left[u_{i, \min }, u_{i, \max }\right] \subset \mathbb{R}^{m} .
\end{aligned}
$$

The right-hand side of the state equation is of the form

$$
f(x, u)= \begin{cases}f_{1}(x, u), & \text { if } S(x, u) \leq 0, \\ f_{2}(x, u), & \text { if } S(x, u)>0,\end{cases}
$$

where the functions $S, f_{k}(k=1,2)$, and $r$ are assumed to be sufficiently smooth.

\section{NECESSARY CONDITIONS}

Let $\left(x^{0}, u^{0}\right)$ denotes a solution of OCP which satisfies the order-zero condition, i.e. $S_{u}\left(x^{0}(t), u^{0}(t)\right) \neq 0$. Further, the following regularity assumption may hold. There exists a finite grid $a=: t_{0}<t_{1}<\ldots<t_{s}<t_{s+1}:=b$ such that the optimal switching function $S[t]$ is either positive or negative in each open subinterval $] t_{j-1}, t_{j}[, \quad j=1, \ldots, s+1$. Then the following necessary conditions hold

Theorem 1 There exist an adjoint variable $\lambda$ : $[a, b] \rightarrow \mathbb{R}^{n}$, which is a piecewise $\mathrm{C}^{1}$-function, and Lagrange multipliers $\nu_{0} \in\{0,1\}, \quad \nu \in \mathbb{R}^{\ell}$, such that

$$
\begin{aligned}
& \lambda^{\prime}(t)=-H_{x}\left(x^{0}(t), u^{0}(t), \lambda(t)\right), \\
& u^{0}(t)=\operatorname{argmin}\left\{H\left(x^{0}(t), u, \lambda(t)\right): u \in U\right\}, \\
& \lambda(a)=-\frac{\partial}{\partial x^{0}(a)}\left[\nu^{T} r\left(x^{0}(a), x^{0}(b)\right)\right], \\
& \lambda(b)=\frac{\partial}{\partial x^{0}(b)}\left[\nu_{0} g\left(x^{0}(b)\right)+\nu^{T} r\left(x^{0}(a), x^{0}(b)\right)\right], \\
& \lambda\left(t_{j}^{+}\right)=\lambda\left(t_{j}^{-}\right), \quad j=1, \ldots, s, \\
& H\left[t_{j}^{+}\right]=H\left[t_{j}^{-}\right], \quad j=1, \ldots, s .
\end{aligned}
$$

Here, the Hamiltonian is given by $H:=H_{k}:=$ $\lambda^{T} f(x, u)$, where $k \in\{1,2\}$ is chosen according to the sign of $S$ in the corresponding subinterval.

If one allows a singular state subarc, say $\left[t_{1}, t_{2}\right]$, which is characterized by $S(x, u)=0$, 
$\forall t \in\left[t_{1}, t_{2}\right]$, on this subarc the minimum principle has to be modified as a constrained minimum principle

$u^{0}(t)=\operatorname{argmin}\left\{H: u \in U, S\left(x^{0}(t), u\right)=0\right\}$.

Further, if the order-zero condition is substituted by the order-one assumption $S=S(x)$ and

$$
S_{u}^{(1)}\left(x^{0}(t), u^{0}(t)\right) \neq 0,
$$

where $S^{(1)}:=S_{x}(x)^{\mathrm{T}} f_{1}(x, u)$, the continuity of the adjoint variable is lost. Instead the following jump condition of the adjoints has to be satisfied

$$
\lambda\left(t_{j}^{+}\right)=\lambda\left(t_{j}^{-}\right)+\kappa_{j} S_{x}\left(x^{0}\left(t_{j}\right)\right), j \in J_{\text {reg }} \cup J_{\text {entry }},
$$

Here, $j \in J_{\text {reg }}$ are the indices of isolated roots $t_{j}$ of the switching function, and $J_{\text {entry }}$ denotes the indices of the entry points of singular state subarcs.

\section{APPLICATIONS}

We consider two examples. The first one is taken from the well-known book of Clarke, Ref. (1). It describes the control of an electronic circuit which encludes a diode and a condensor. The control $u$ is the initializing voltage, the state variable $x$ denotes the voltage at the condensor. The problem is given in form of a nonsmooth OCP of the order zero. Depending on the parameters of the model, the solution may contain a singular state subarc.

The second example is an economic optimal control model due to Pohmer, Ref. (2),(3), which describes the personal income distribution of a typical consumer, who wants to maximize the total utility of his lifetime by controling the consumption, the rate of the total time used for working, and the rate of working time used for education and extended professional training. The state variables are the human capital and the capital itself. The utility function contains different parts which represent the influence of consumption, time of recreation, and human capital. Into this problem a parameter enters which describes the interest rate of capital. It is obvious that this parameter in general will differ for positive and negative values of the capital. Thus, the resulting problem in a natural way becomes a nonsmooth
OCP of the order one. For this, the necessary conditions are derived and numerical solutions are presented. Again, it turns out that for a certain distance of the positive and negative interest rate, the optimal solution contains a singular state subarc.

\section{REFERENCES}

[1] Clarke F.H. Optimization and Nonsmooth Analysis. Wiley: New York, 1983.

[2] Pohmer K. Mikroökonomische Theorie der personellen Einkommens- und Vermögensverteilung. Studies in Contemporary Economics, Vol. 16, Springer: 1985.

[3] Oberle, H.J. and R. Rosendahl Numerical computation of a singular-state subarc in an economic optimal control problem. Optimal Control Application and Methods, 27, 211-235, 2006.

[4] Oberle, H.J. Nonsmooth Optimal Control Problems with Switching Functions of Order Zero. Preprint 192, Hamburger Beiträge zur Angewandten Mathematik, University of Hamburg, 2006. 